\title{
An Intelligent DNA Nanorobot with in vitro Enhanced Protein Lysosomal
}

Wenjuan Ma, ${ }^{\dagger}$ Yuxi Zhan, ${ }^{\dagger}$ Yuxin Zhang, ${ }^{\dagger}$ Xiaoru Shao, ${ }^{\dagger}$ Xueping Xie, ${ }^{\dagger}$ Chenchen Mao, ${ }^{\dagger}$

Weitong Cui, ${ }^{\dagger}$ Qian Li, ${ }^{\ddagger}$ Jiye Shi, ${ }^{\S}$ Jiang Li, ${ }^{\ddagger}$ Chunhai Fan, $\stackrel{\ddagger}{\S}$ Yunfeng Lin $*, \dagger$

$\dagger$ State Key Laboratory of Oral Diseases, National Clinical Research Center for Oral Diseases,

West China Hospital of Stomatology, Sichuan University, Chengdu 610041, P. R. China.

$¥$ School of Chemistry and Chemical Engineering, and Institute of Molecular Medicine, Renji

Hospital, School of Medicine, Shanghai Jiao Tong University, Shanghai 200240, China.

§ Division of Physical Biology and Bioimaging Center, Shanghai Synchrotron Radiation

Facility, CAS Key Laboratory of Interfacial Physics and Technology, Shanghai Institute of

Applied Physics, Chinese Academy of Sciences, Shanghai 201800, China

*Correspondence Author: Yunfeng Lin

State Key Laboratory of Oral Diseases, National Clinical Research Center for Oral Diseases,

19 West China Hospital of Stomatology, Sichuan University, Chengdu 610041, P. R. China

Tel: 86-28-85503487; Fax: 86-28-85503487

21 Email address: yunfenglin@scu.edu.cn 


\section{Characterization of Single-stranded DNAs.}

2 The characterization of all single-stranded DNAs (ssDNAs) was confirmed by 3 polyacrylamide gel electrophoresis ( $8 \%$, PAGE). The co-mixtures of each sample (S1, S2, S3,

4 S4, free HApt, HApt-S4) with $6 \times$ loading buffer at a ratio of 5:1 were added to the PAGE gel, 5 and electrophoresis was conducted at a constant voltage of $80 \mathrm{~V}$ for $80 \mathrm{~min}$, dyed by Gel-Red 6 (Sigma) for $20 \mathrm{~min}$, and analyzed by an ultraviolet exposure apparatus (Bio-Rad, Hercules, 7 CA, USA).

8

9 Figure S1. Characterization of ssDNAs by polyacrylamide gel electrophoresis (PAGE). Lane

1, S1. Lane 2, S2. Lane 3, S3. lane 4, S4. Lane 5, free HApt. Lane 6, HApt-S4.

\section{Characterization of HApt-tFNA.}

For identification of the successful assembly of HApt-tFNA, dynamic light scattering (DLS) was used to detect the particle size and the polymer dispersion index (PDI) of the nanostructures (tFNA and HApt-tFNA) with a Zetasizer Nano ZS90 system (Malvern Instruments Ltd, UK). In addition, transmission electron microscopy (TEM, Libra 200, Zeiss,

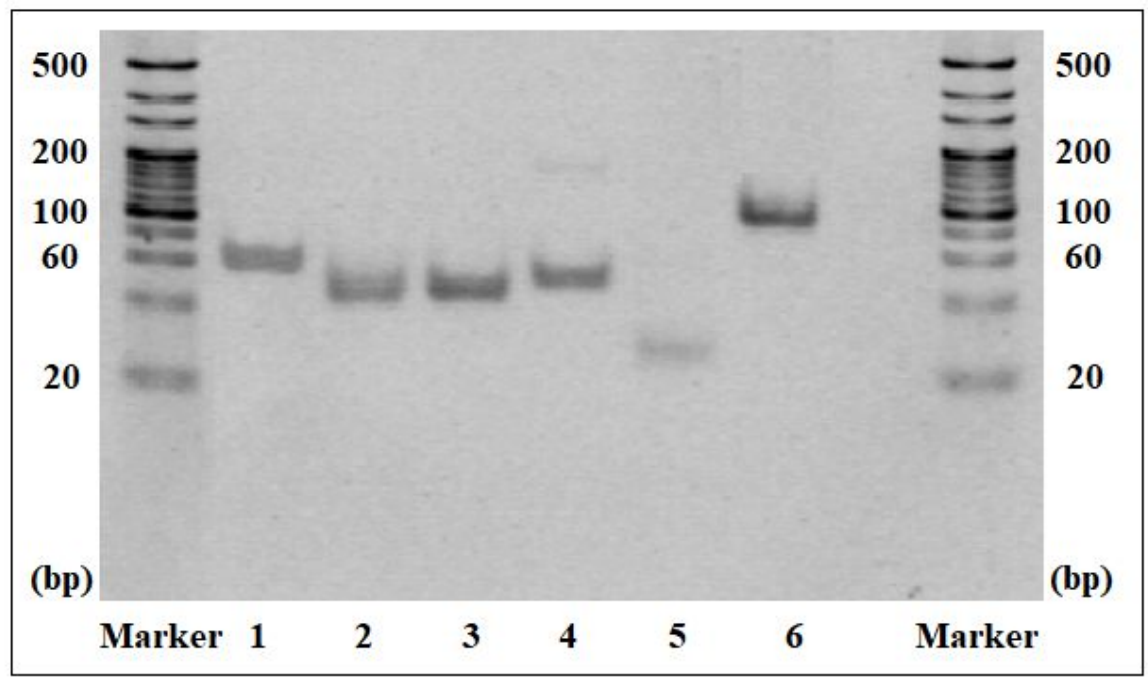


1 Germany) was applied to measure the size of the HApt-tFNA, and the images of HApt-tFNA

2 were obtained without requiring a special stain. Besides, atomic force microscope (AFM, SPM-

39700 instrument, Shimadzu, Kyoto, Japan) was applied to observe the morphology of tFNA

4 and HApt-tFNA. Before we conducted the AFM, the tFNA and HApt-tFNA were dropped on

5 a cleaved mica, air-dried for 20 min, and tested by AFM. The size of tFNA and HApt-tFNA

6 was $11.305 \pm 0.782 \mathrm{~nm}$ and $19.818 \pm 0.862 \mathrm{~nm}$ by DLS, respectively (Figure S2.A and Figure

7 S2.B). As shown in Figure S2.C, the PDI of tFNA was $0.424 \pm 0.105$, and that of HApt-tFNA

8 was $0.667 \pm 0.115$. This size difference indicated the successful formation of HApt-tFNA. The

9 results of TEM showed that the HApt-tFNA were approximately $20.0 \mathrm{~nm}$ (Figure S3). As the

10 AFM results shown in Figure S4, the size of tFNA was approximately $10.0 \mathrm{~nm}$, and the height

11 was about $2.0 \mathrm{~nm}$, while the size and height of HApt-tFNA were approximately $20.0 \mathrm{~nm}$ and

$123.0 \mathrm{~nm}$, respectively. From the results of AFM, we could further ensure that the nanorobot

13 (HApt-tFNA) was generated successfully.
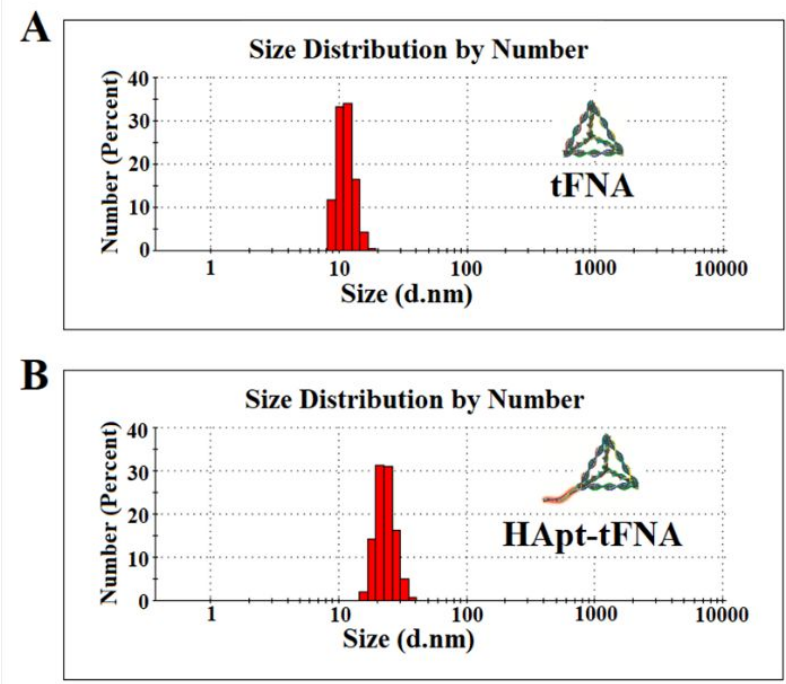

\begin{tabular}{c|c|c}
\hline & tFNA & HApt-tFNA \\
\hline Size (d.nm) & $11.305 \pm 0.782$ & $19.818 \pm 0.862$ \\
\hline PDI & $0.424 \pm 0.105$ & $0.667 \pm 0.115$ \\
\hline
\end{tabular}


1 Figure S2. Dynamic light scattering (DLS) measurement statistics of the difference between

2 tFNA and HApt-tFNA. A) The size distribution results of tFNA. B) The size distribution results 3 of HApt-tFNA. C) The size and PDI results of tFNA and HApt-tFNA.

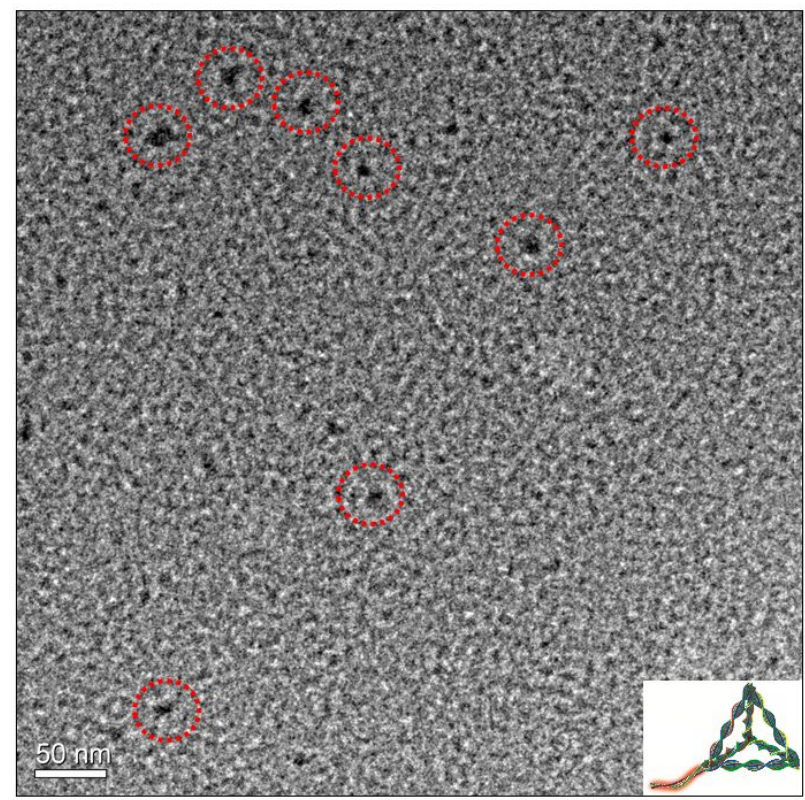

6 Figure S3. Transmission electron microscopy (TEM) image of HApt-tFNA (HApt-tFNA: red circle). Scale bars are $50 \mathrm{~nm}$.

8

A

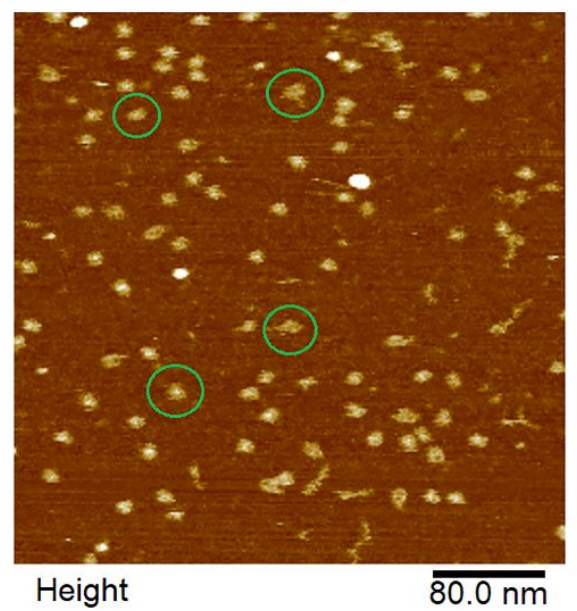

B

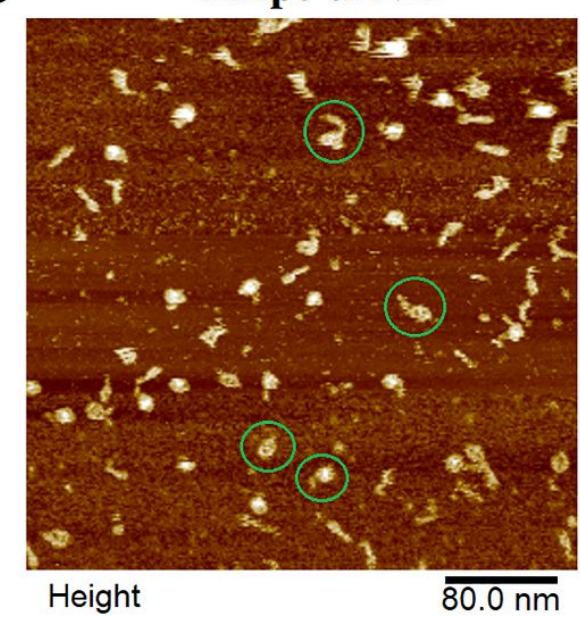

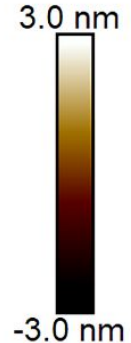

$-3.0 \mathrm{~nm}$

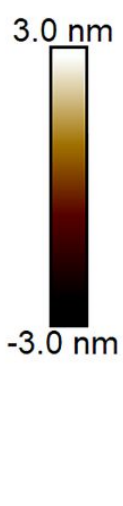

Figure S4. Atomic force microscope (AFM) images of tFNA and HApt-tFNA. A) The AFM 
1 image of tFNA (tFNA: green circle). Scale bars are $80 \mathrm{~nm}$. B) The AFM image of HApt-tFNA

2 (HApt-tFNA: green circle). Scale bars are $80 \mathrm{~nm}$.

\section{Plasma Half-time Measurement}

In order to observe the half-life of HApt-tFNA and free HApt, we injected free Cy5-HApt (1 $\mu \mathrm{M} ; 100 \mu \mathrm{L} /$ mouse)/Cy5-HApt-tFNA ( $1 \mu \mathrm{M} ; 100 \mu \mathrm{L} /$ mouse) into the tail vein of 6-week-

7 old nude mice (20g; male; Balb/c). Blood (5 $\mu \mathrm{M} /$ mouse) was harvested from tail vein at

8 indicated time points ( $2 \mathrm{~min}, 5 \mathrm{~min}, 10 \mathrm{~min}, 20 \mathrm{~min}, 30 \mathrm{~min}, 45 \mathrm{~min}, 60 \mathrm{~min}, 90 \mathrm{~min}, 120 \mathrm{~min}$ ),

9 deliquated 100-fold in phosphate-buffered saline (PBS, Gibco) containing EDTA (5 mM). All

10 blood samples were centrifuged and the fluorescent signal of Cy5 in the supernatants were

11 quantified by a fluorescent microplate reader (SpectraMax i3X, Molecular Devices, USA). The

12 fluorescent intensity of Cy5 at different time points was normalized to the 2-min value as the

13 residual free Cy5-HApt or Cy5-HApt-tFNA in the circulation. As shown in Figure S5, the half-

14 life of free HApt was about $15 \mathrm{~min}$, and that of HApt-tFNA was 35 min approximately. The

15 significant difference between free HApt and HApt-tFNA indicated that HApt-tFNA had a

16 longer half-life than free HApt, which was critical for therapeutic efficacy.

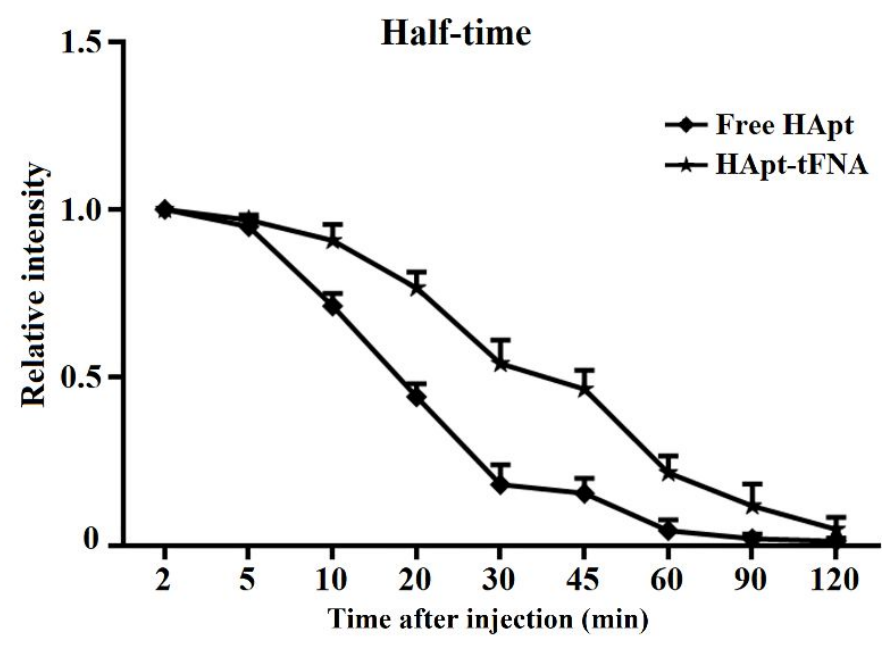

Page S1/ S19 
1 Figure S5. Plasma half-time measurement. The free HApt and HApt-tFNA were labeled with

2 Cy5. The free Cy5-HApt and Cy5-HApt-tFNA were injected into mice respectively. ( $n=$

34 /group). Data is presented as mean $\pm \mathrm{SD}(n=4)$.

4

\section{Immunoblotting of HER2 in Different Cells.}

6

After treating cells (SK-BR-3, MCF-7, and MCF-10A cells) cultured for $24 \mathrm{~h}$, total proteins

7 were harvested using Whole Cell Lysis Assay (KeyGEN Biotech), mixed with $5 \times$ loading

8 buffer at a ratio of $4: 1$, and heated at $100^{\circ} \mathrm{C}$ for $10 \mathrm{~min}$. All protein samples were subjected to

9 SDS-PAGE (Beyotime, Nanjing, China), diverted to a polyvinylidene fluoride membrane

10 (TaKaRa), and blocked with $5 \%$ bovine serum albumin (Sigma) solution for $1 \mathrm{~h}$ at $37^{\circ} \mathrm{C}$.

11 Various primary antibodies (anti-GAPDH, and anti-ErbB2; 1:1000; Abcam) were incubated

12 with corresponding membranes overnight at $4{ }^{\circ} \mathrm{C}$. The next day, the membranes were rewashed

with TBST (100 mM NaCl,10 mM Tris-base, and 0.1\% Tween-20; $\mathrm{pH} 7.5$ ) three times,

exposed to secondary antibodies $(1: 2000)$ for $1 \mathrm{~h}$ at $37^{\circ} \mathrm{C}$, and visualized using an enhanced

chemiluminescence detection system (Bio-Rad). The internal reference was GAPDH. We

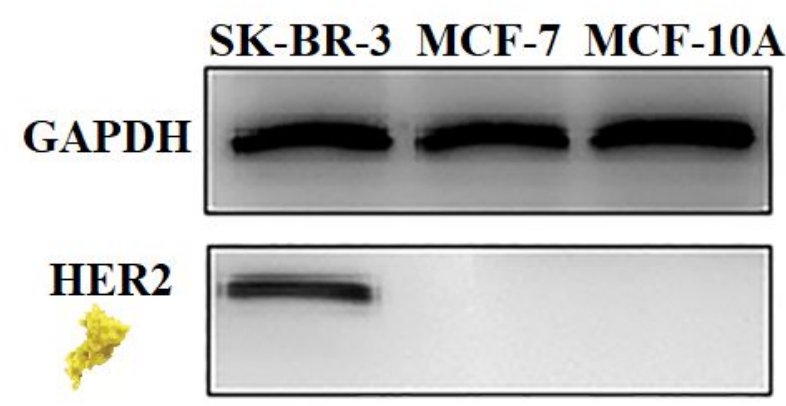

19 Figure S6. The expression of HER2 in different cells (SK-BR-3, MCF-7 and MCF-10A cells) 
by immunoblotting analysis.

\section{Cell Viability Measurement.}

4

To determine the optimal concentration of HApt-tFNA and free HApt, all cells were exposed

to various concentrations $(62.5 \mathrm{nM}, 125 \mathrm{nM}, 250 \mathrm{nM}, 375 \mathrm{nM})$ and cell viability was assessed

6 with the reagent (CCK-8; Dojindo Laboratories, Japan). SK-BR-3, MCF-7, and MCF-10A

cells were cultured in 96 -well plates $\left(5 \times 10^{4}\right.$ cells $\left./ \mathrm{mL}\right)$, and then exposed to various

concentrations of free HApt and HApt-tFNA the next day for $24 \mathrm{~h}$ and $48 \mathrm{~h}$, respectively.

9 Medium $(100 \mu \mathrm{L})$ was mixed with CCK-8 reagent $(10 \mu \mathrm{L})$ per well, and a group of wells was

10 treated with only medium as the control for background subtraction. After $1-4 \mathrm{~h}$ incubation,

the absorbance of samples was measured by a Varioskan Flash microplate reader (Thermo

Scientific, Waltham, MA, USA). We found that HApt-tFNA at $250 \mathrm{nM}$ could reduce the viability of SK-BR-3 cells effectively after treatment from 24 h to 48 h (Figure S7), whereas the viability of MCF-7 and MCF-10A cells was increased initially, and then was not affected at $48 \mathrm{~h}$. Exposure of MCF-10A cells to $375 \mathrm{nM}$ HApt-tFNA was harmful at $48 \mathrm{~h}$.
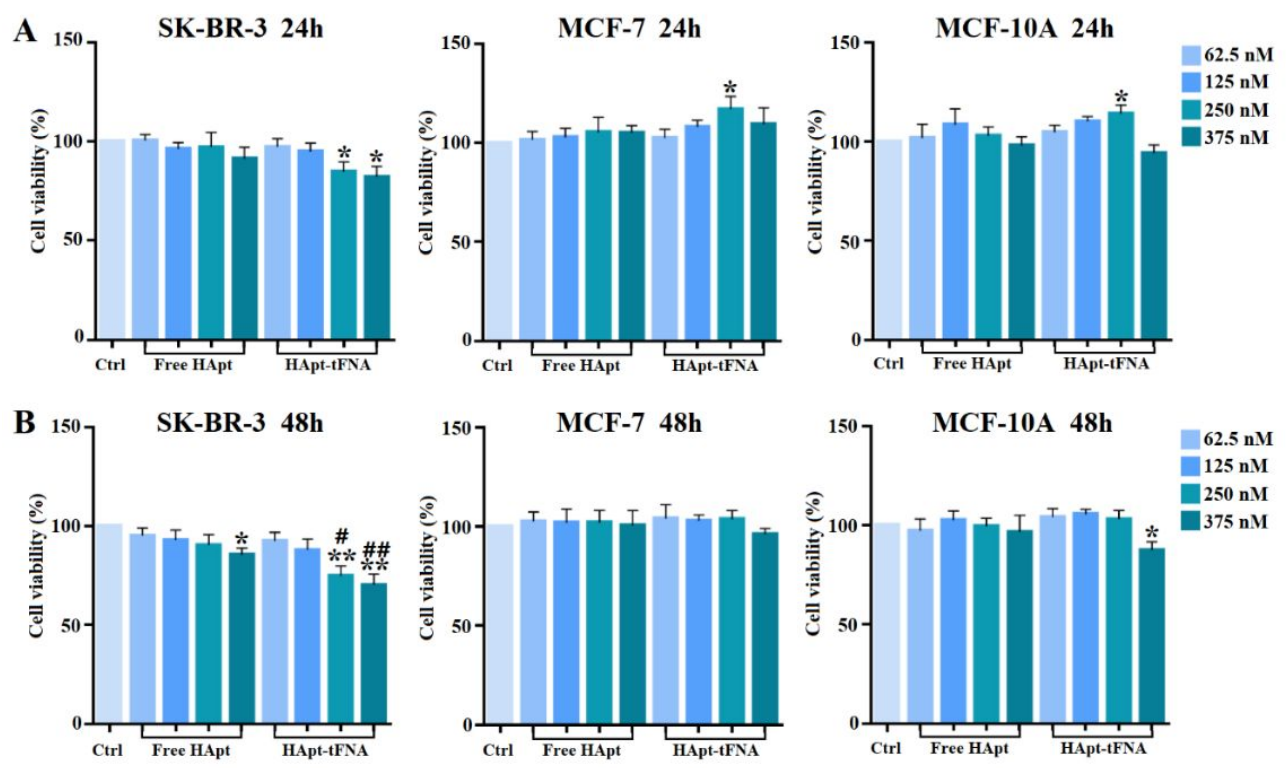

Page S1/ S19 
1 Figure S7. Cell viability assay (CCK-8 assay) of SK-BR-3, MCF-7 and MCF-10A cells. A)

2 Cell viability assay (CCK-8 assay) of SK-BR-3, MCF-7 and MCF-10A cells after treated with

3 free HApt and HApt-tFNA for $24 \mathrm{~h}$. Data is presented as mean $\pm \mathrm{SD}(n=4)$. Statistical analysis:

$4 * p<0.05$.vs. Control. B) Cell viability assay (CCK-8 assay) of SK-BR-3, MCF-7 and MCF-

5 10A cells after treated with free HApt and HApt-tFNA for $48 \mathrm{~h}$. Data is presented as mean \pm

$6 \quad \mathrm{SD}(n=4)$. Statistical analysis: ${ }^{*} p<0.05 . v s$. Control; ${ }^{* *} p<0.01 . \mathrm{vs}$. Control; ${ }^{\#} p<0.05 . \mathrm{vs}$.

7 free HApt with the same concentration; ${ }^{\#} p<0.01$.vs. free HApt with the same concentration.

8

6. Cell Viability Measurement of SK-BR-3 Cells Treated with 250 nM tFNA.

10 SK-BR-3 cells were cultured in 96-well plates $\left(5 \times 10^{3}\right.$ cells $\left./ \mathrm{mL}\right)$, and then exposed to 250

$11 \mathrm{nM}$ tFNA the next day for $24 \mathrm{~h}, 48 \mathrm{~h}$, and $72 \mathrm{~h}$, respectively. Medium (100 $\mu \mathrm{L})$ was mixed with

12 CCK-8 reagent $(10 \mu \mathrm{L})$ per well, and a group of wells was treated with only medium as the

control for background subtraction. After $1-4 \mathrm{~h}$ incubation, the absorbance of samples was

measured by a Varioskan Flash microplate reader (Thermo Scientific, Waltham, MA, USA).

As shown in Figure S8, the viability of SK-BR-3 cells exposed to $250 \mathrm{nM}$ tFNA was increased initially, and then was not affected at $48 \mathrm{~h}$ or $72 \mathrm{~h}$.

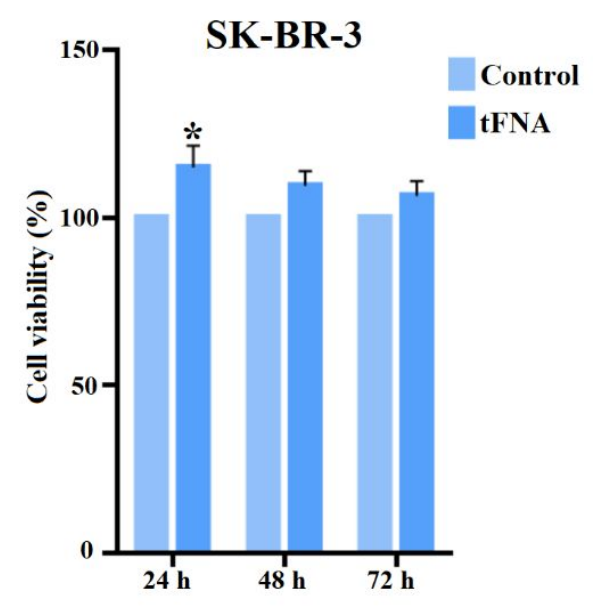


1 Figure S8. Cell viability measurement of SK-BR-3 cells treated with $250 \mathrm{nM}$ tFNA for $24 \mathrm{~h}$,

$248 \mathrm{~h}$, and $72 \mathrm{~h}$. Data is presented as mean $\pm \mathrm{SD}(n=3)$. Statistical analysis: $* p<0.05 . \mathrm{vs}$.

3 Control.

4

7. Cy5-Labeled Free HApt and HApt-tFNA Cellular Uptake Assay.

To analyze the difference in cellular uptake between free Cy5-HApt and Cy5-HApt-tFNA,

7 flow cytometry was applied ${ }^{1}$. For flow cytometry analyses, SK-BR-3, MCF-7, and MCF-10A

8 cells were seeded into plates and treated with growth medium containing $250 \mathrm{nM}$ free Cy5-

9 HApt or Cy5-HApt-tFNA for $12 \mathrm{~h}$. After rinsing with PBS three times, a flow cytometer

10 (FC500 Beckman, IL, USA) was used to analyze the cellular uptake of free Cy5-HApt and

11 Cy5-HApt-tFNA. As shown in Figure S9, more than 60\% of the SK-BR-3 cells took in Cy5-

12 HApt-tFNA, while only about $12 \%$ of the SK-BR-3 cells took in free Cy5-HApt. The HApt-

13 tFNA could be internalized by SK-BR-3 cells much more obviously than free HApt, which

14 might be also be related to the higher stability of HApt-tFNA and the ability of tFNA to drive

15 the nanostructures into cells. By contrast, only a few MCF-7 (less than 4\%) and MCF-10A

16 cells (less than 10\%) could assimilate free Cy5-HApt or Cy5-HApt-tFNA. Therefore, both free

17 Cy5-HApt and Cy5-HApt-tFNA could target and enter SK-BR-3 cells by recognizing HER2

specifically, although Cy5-HApt-tFNA showed much better targeting ability. 

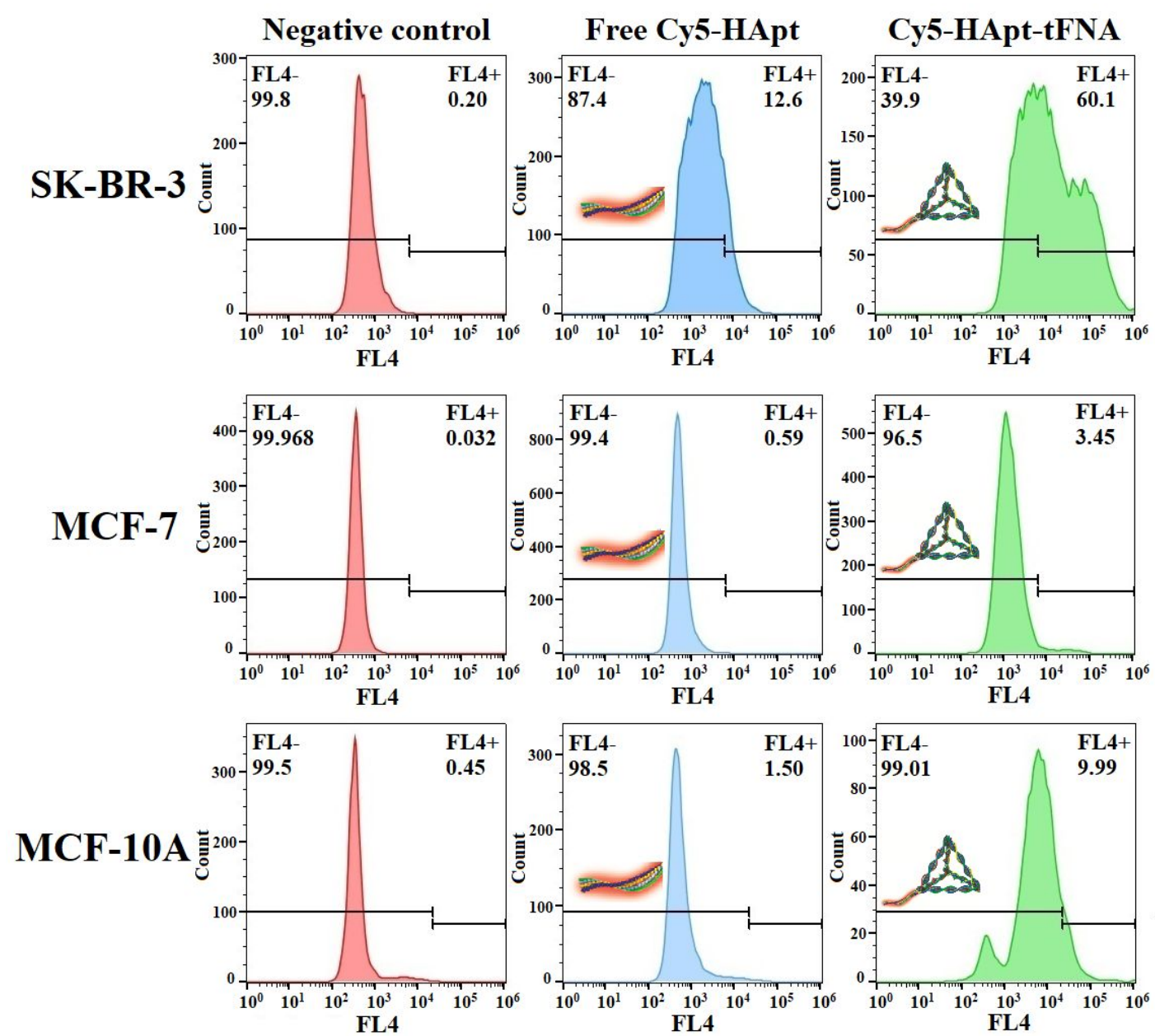

2 Figure S9. Flow cytometry analysis of the cellular uptake rates in SK-BR-3, MCF-7 and MCF-

3 10A cells incubated with free Cy5-HApt or Cy5-HApt-tFNA for $12 \mathrm{~h}$.

\section{Cellular Uptake of tFNA by Different Cells.}

To analyze cellular uptake of tFNA by difference cells, confocal imaging and flow cytometry were applied ${ }^{1}$. SK-BR-3, MCF-7, and MCF-10A cells were seeded on confocal plates and

8 cultured in their respective complete growth media. After $24 \mathrm{~h}$, the cells were treated with fresh

9 growth medium containing $250 \mathrm{nM}$ Cy5-tFNA for $12 \mathrm{~h}$, and then residual nanostructures were

10 removed. All cells were fixed in cold 4\% paraformaldehyde (Gibco) for 20 min, permeated by

$110.05 \%$ Triton-X100 for $10 \mathrm{~min}$, stained by phalloidin (Sigma) for $1 \mathrm{~h}$ at $37^{\circ} \mathrm{C}$, and treated with 
1 DAPI (Sigma) for $10 \mathrm{~min}$. All cells were examined under a confocal laser-scanning microscope

2 (Nikon N-SIM) to determine the cellular uptake of Cy5-tFNA. For flow cytometry analyses,

3 all cells were seeded into plates and treated with growth medium containing $250 \mathrm{nM} \mathrm{Cy} 5$-tFNA

4 for $12 \mathrm{~h}$. After rinsing with PBS three times, a flow cytometer (FC500 Beckman, IL, USA) was

5 used to analyze the cellular uptake of free Cy5-tFNA. As shown in Figures S10, S11, and S12,

6 we could find that tFNA could enter cells (SK-BR-3, MCF-7, and MCF-10A cells) ${ }^{2-8}$.
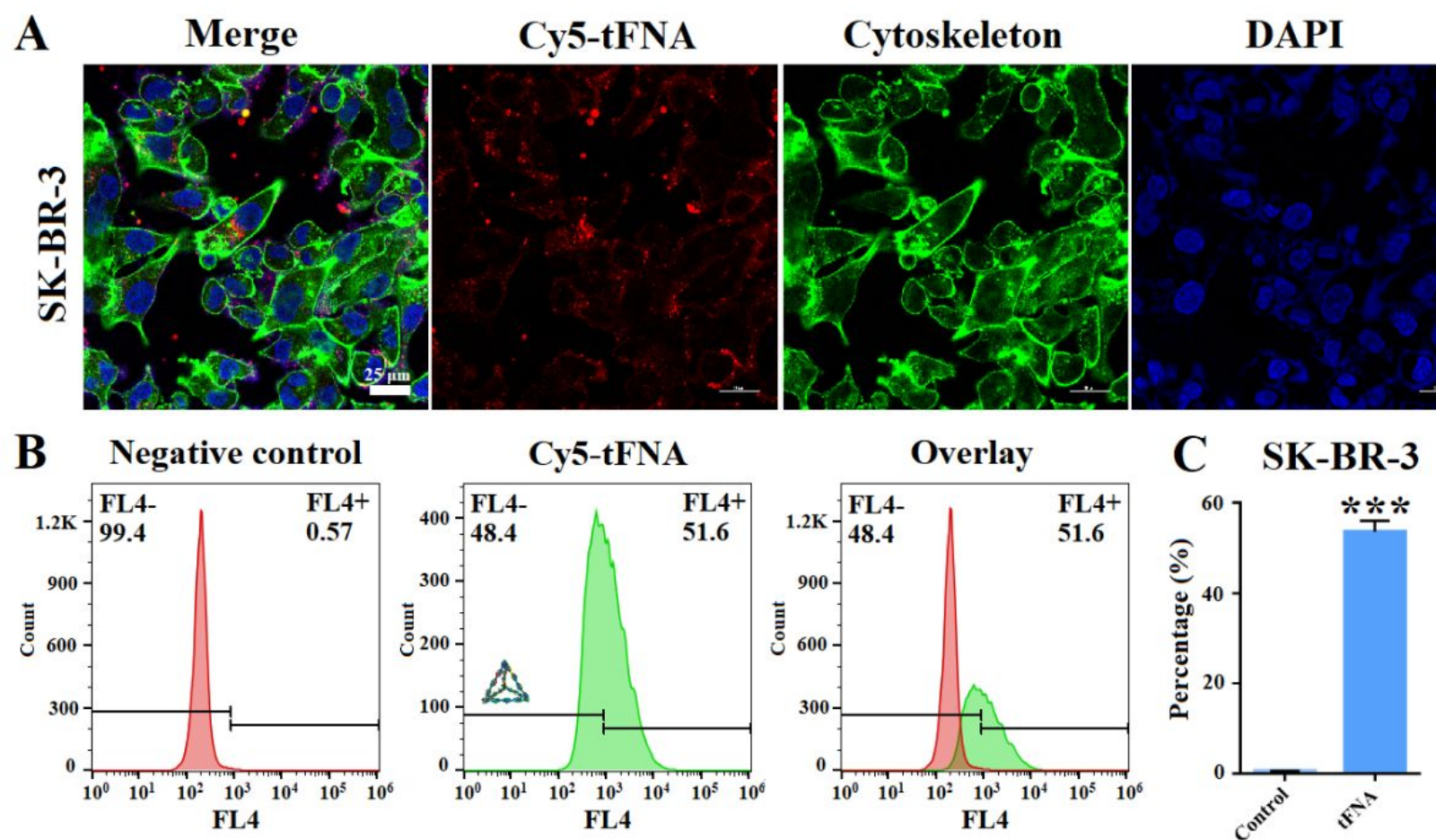

Figure S10. Cellular uptake of Cy5-tFNA by SK-BR-3 cells. A) Confocal laser scanning

9 microscopy images show that cellular uptake of Cy5-tFNA in SK-BR-3 cells (12 h of

10 incubation; Cy5-tFNA: red, cytoskeleton: green, nucleus: blue). Scale bars are $25 \mu \mathrm{m}$. B) Flow

11 cytometry analysis of the uptake rates in SK-BR-3 cells incubated with Cy5-tFNA for 12 h. C)

12 Quantitative analysis of cellular uptake rates in SK-BR-3 cells treated with Cy5-tFNA for 12

h. Data is presented as mean $\pm \operatorname{SD}(n=3)$. Statistical analysis: $* * * p<0.001$.vs. Control. 

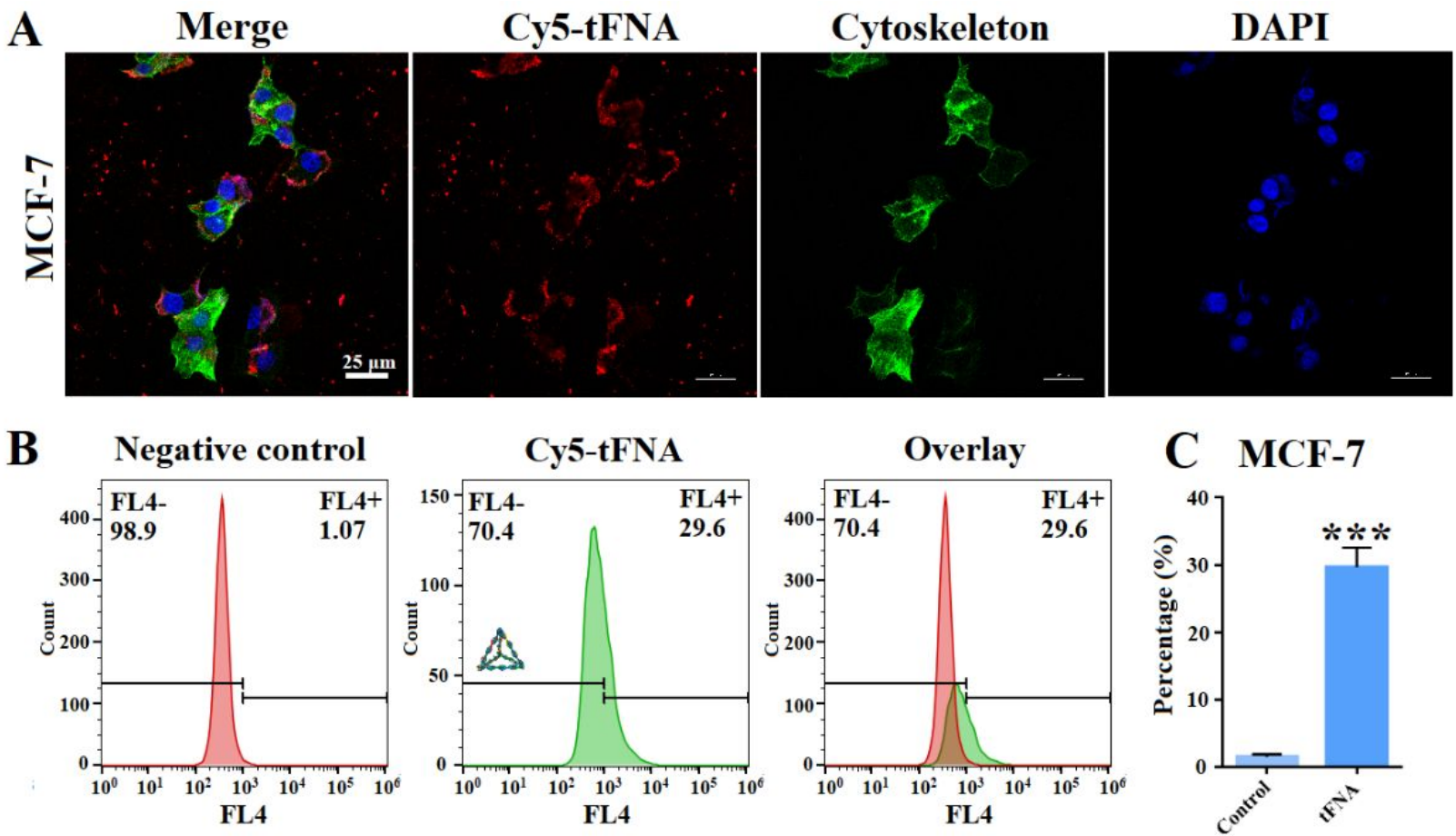

C $\quad$ MCF-7

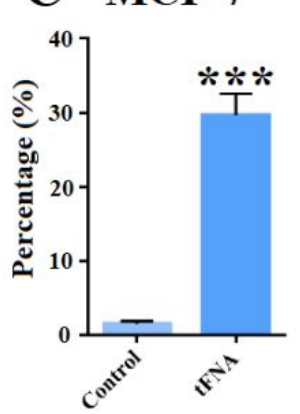

2 Figure S11. Cellular uptake of Cy5-tFNA by MCF-7 cells. A) Confocal laser scanning

3 microscopy images show that cellular uptake of Cy5-tFNA in MCF-7 cells (12 h of incubation;

4 Cy5-tFNA: red, cytoskeleton: green, nucleus: blue). Scale bars are $25 \mu \mathrm{m}$. B) Flow cytometry

5 analysis of the uptake rates in MCF-7 cells incubated with Cy5-tFNA for $12 \mathrm{~h}$. C) Quantitative

6 analysis of cellular uptake rates in MCF-7 cells treated with Cy5-tFNA for $12 \mathrm{~h}$. Data is

7 presented as mean $\pm \mathrm{SD}(n=3)$. Statistical analysis: $* * * p<0.001 . v s$. Control. 

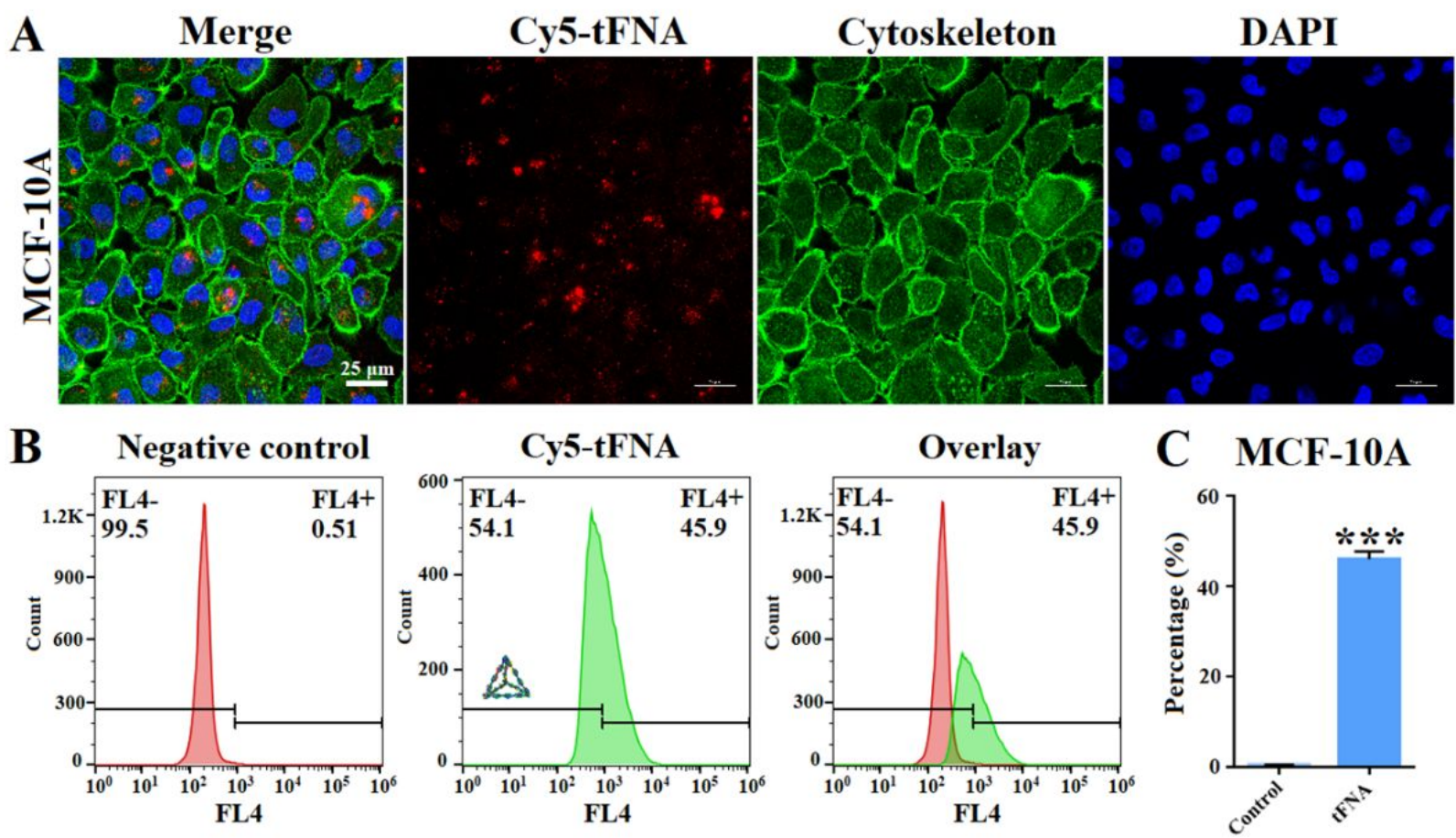

2 Figure S12. Cellular uptake of Cy5-tFNA by MCF-10A cells. A) Confocal laser scanning

3 microscopy images show that cellular uptake of Cy5-tFNA in MCF-10A cells (12 h of

4 incubation; Cy5-tFNA: red, cytoskeleton: green, nucleus: blue). Scale bars are $25 \mu \mathrm{m}$. B) Flow

5 cytometry analysis of the uptake rates in MCF-10A cells incubated with Cy5-tFNA for $12 \mathrm{~h}$.

6 C) Quantitative analysis of cellular uptake rates in MCF-10A cells treated with Cy5-tFNA for

$712 \mathrm{~h}$. Data is presented as mean $\pm \mathrm{SD}(n=3)$. Statistical analysis: $* * * p<0.001 . v s$. Control.

\section{Effects of HApt-tFNA on Cell Biological Behaviors of MCF-7 and MCF-10A Cells.}

Flow cytometry was conducted to observe the effect of HApt-tFNA on the cell cycle and cell apoptosis of MCF-7 and MCF-10A cells. For cell cycle analysis, MCF-7 and MCF-10A cells were treated with $250 \mathrm{nM}$ free HApt or HApt-tFNA for $48 \mathrm{~h}$, and then were collected, rinsed with PBS twice, and fixed in cold $70 \%$ ethanol overnight at $-20^{\circ} \mathrm{C}$. The next day, the cells were washed in PBS three times, treated with $100 \mu \mathrm{L}$ RNase A (30 min at $37^{\circ} \mathrm{C}$ ), and then 
1 exposed to $400 \mu \mathrm{L}$ propidium iodide (PI; 30 min at $4^{\circ} \mathrm{C}$ ). Finally, the cell cycle was examined on an FC500 Beckman flow cytometer. After the same treatment, MCF-7 and MCF-10A cells

3 were harvested and washed thrice with PBS for analysis of cell apoptosis. In brief, the cells

4 were incubated with $400 \mu \mathrm{L}$ Annexin V binding solution stained with $5 \mu \mathrm{L}$ Annexin V-FITC

5 solution $\left(10 \mathrm{~min}\right.$ at $\left.37^{\circ} \mathrm{C}\right)$, followed by $5 \mu \mathrm{L}$ PI solution $\left(10 \mathrm{~min}\right.$ at $\left.4^{\circ} \mathrm{C}\right)$. Ultimately, cell

6 apoptosis was investigated by flow cytometry (FC500 Beckman). We found that HApt-tFNA

7 could increase the number of cells in S phase compared to free HApt both in MCF-7 (Figure

8 S13.A and S13.B) and MCF-10A cells (Figure S14.A and S14.B), but there were no significant

9 differences between the effects of free HApt and HApt-tFNA on cell apoptosis in MCF-7 and

MCF-10A cells (Figure S13.C and S14.C).
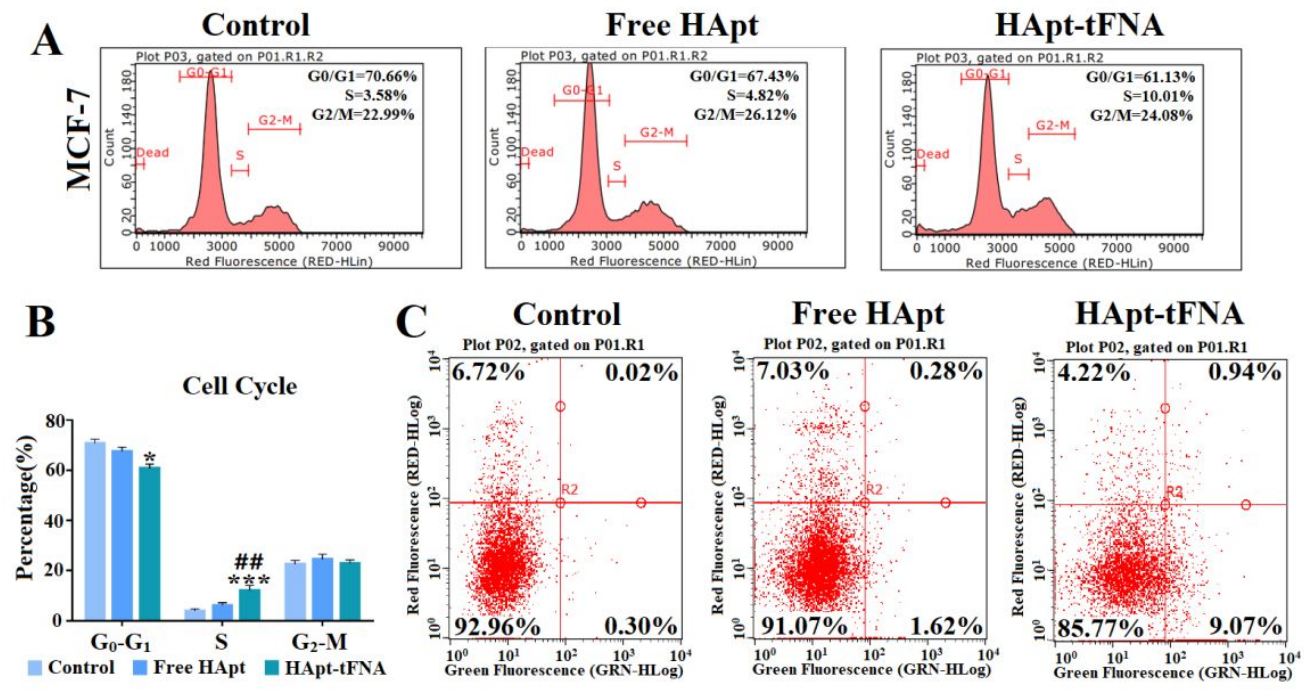

Figure S13. Effects of free HApt and HApt-tFNA on MCF-7 cells. A) Flow cytometry analysis of cell cycle after MCF-7 cells exposed to free HApt or HApt-tFNA for $48 \mathrm{~h}$. B) Data analysis of cell cycle distribution. Data is presented as mean $\pm \mathrm{SD}(n=3)$. Statistical analysis: ${ }^{*} p<$ 0.05.vs. Control; *** $p<0.001 . v s$. Control; ${ }^{\#} p<0.01 . v s$. free HApt. C) Flow cytometry analysis of cell apoptosis after MCF-7 cells exposed to free HApt or HApt-tFNA for $48 \mathrm{~h}$. 

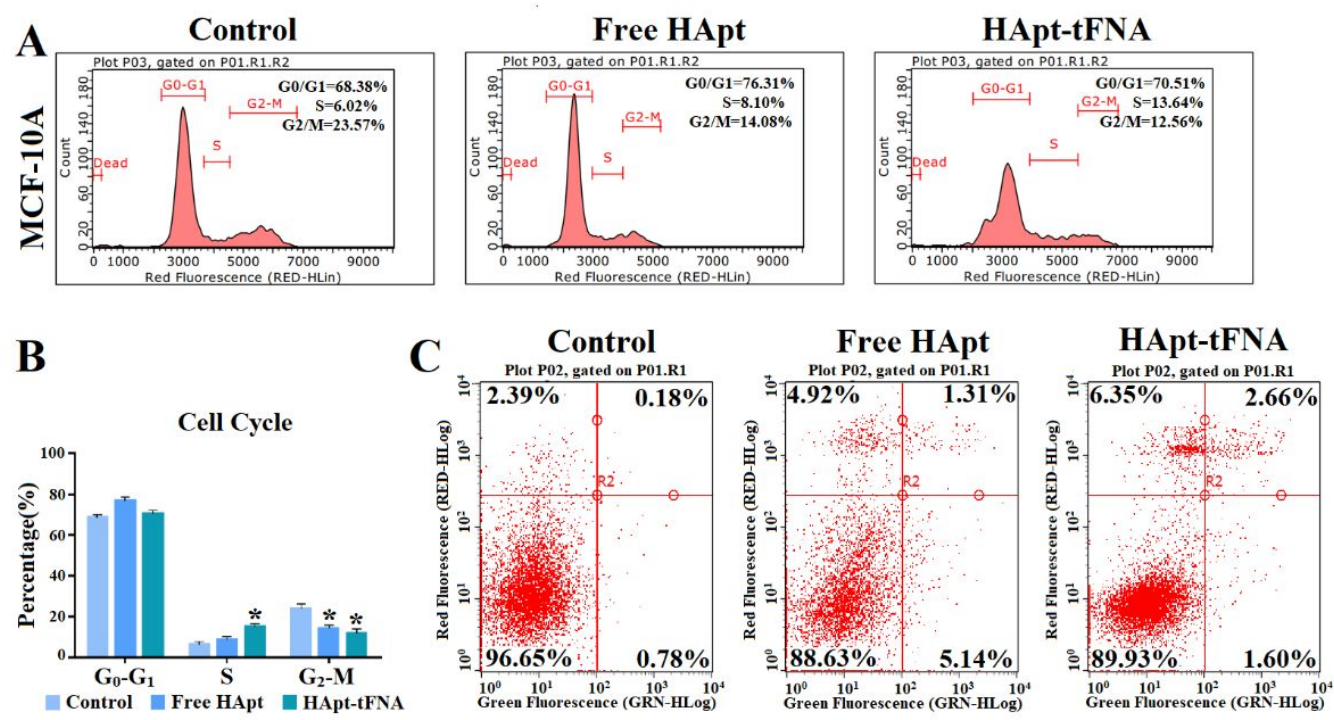

Figure S14. Effects of free HApt and HApt-tFNA on MCF-10A cells. A) Flow cytometry analysis of cell cycle after MCF-10A cells exposed to free HApt or HApt-tFNA for $48 \mathrm{~h}$. B) Data analysis of cell cycle distribution. Data is presented as mean $\pm \mathrm{SD}(n=3)$. Statistical analysis: ${ }^{*} p<0.05$.vs. Control. C) Flow cytometry analysis of cell apoptosis after MCF-10A cells exposed to free HApt or HApt-tFNA for $48 \mathrm{~h}$.

\section{Distribution of Cy5-tFNA in SK-BR-3 cells.}

To observe whether tFNA could be taken into lysosomes in SK-BR-3 cells, Lyso Tracker

12 Green DND26 (Invitrogen) was applied. SK-BR-3 cells were plated on confocal plates for 24 h, and then co-cultured with $250 \mathrm{nM}$ Cy5-tFNA for $12 \mathrm{~h}$, respectively. Next, the growth medium was changed to the medium containing Lyso Tracker Green DND26 at $37^{\circ} \mathrm{C}$ for $1 \mathrm{~h}$. Then, the cells were fixed in cold paraformaldehyde and stained with DAPI. Finally, confocal images were captured by a confocal laser-scanning microscope (Nikon N-SIM). After 
1 incubated with free Cy5-tFNA for $12 \mathrm{~h}$, we found the fluorescence signals of Cy5 showed high 2 uptake by SK-BR-3 cells and clustering in lysosomes, indicating that tFNA could enter the 3 lysosomes of SK-BR-3 cells (Figure S15).

4
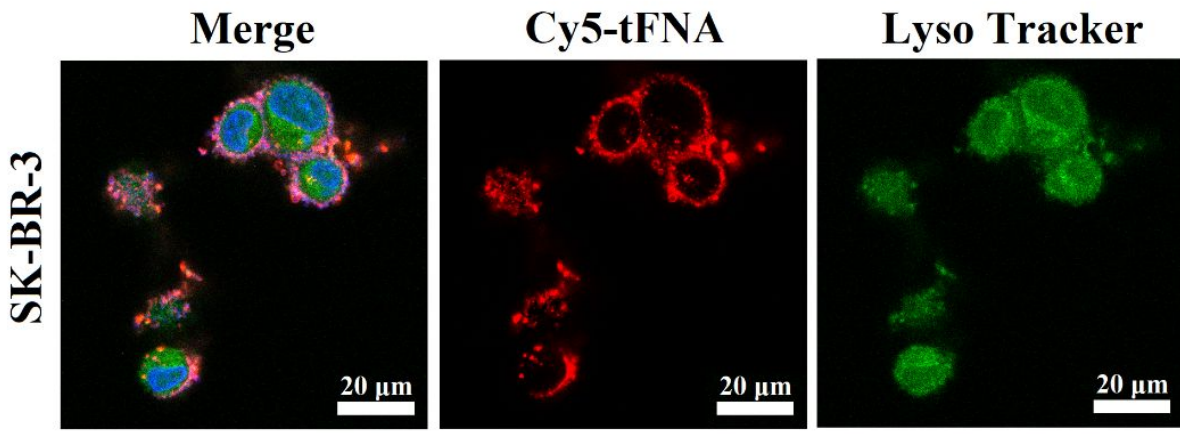

DAPI

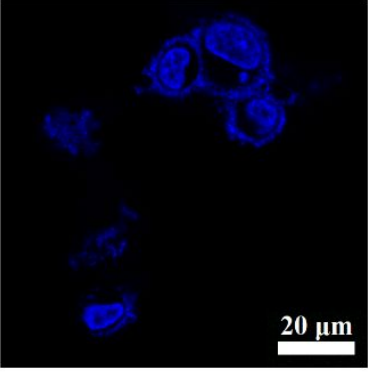

Figure S15. Distribution of Cy5-tFNA in SK-BR-3 cells. After incubated with free Cy5-tFNA for $12 \mathrm{~h}$, the fluorescence signals of Cy5 showed high uptake by SK-BR-3 cells and clustering in lysosomes (Cy5-tFNA: red, lysosome: green, nucleus: blue). Scale bars are $20 \mu \mathrm{m}$.

\section{The Observation of HER2-Cy5-HApt-tFNA Complexes.}

The SK-BR-3 cells were cultured on confocal plates with their own complete growth medium for $24 \mathrm{~h}$. After cultured with Cy5-HApt-tFNA for $24 \mathrm{~h}$, all cells were washed with PBS three times to remove residual growth medium. All samples were then immobilized by $4 \%$ paraformaldehyde for $20 \mathrm{~min}$, permeabilized by $0.05 \%$ Triton-X100 (Gibco) for $10 \mathrm{~min}$, and blocked with $5 \%$ sheep serum (Corning) for $1 \mathrm{~h}$ at $37^{\circ} \mathrm{C}$. All cells were incubated with primary antibody (rabbit anti-ErbB2, 1:250 dilution; Abcam, Cambridge, UK) at $4^{\circ} \mathrm{C}$ overnight, and then with goat anti-rabbit IgG (1:1000 dilution; Abcam) for $1 \mathrm{~h}$. The cell nuclei were treated with DAPI for 10 min. All confocal images were acquired with a confocal laser-scanning microscope (Nikon N-SIM, Japan). When we observed the results of Figure 5C, we found the HER2-Cy5-HApt-tFNA complex in cells fortunately. The Figure S16 was extracted from the 
1 results of Figure 5C.

2
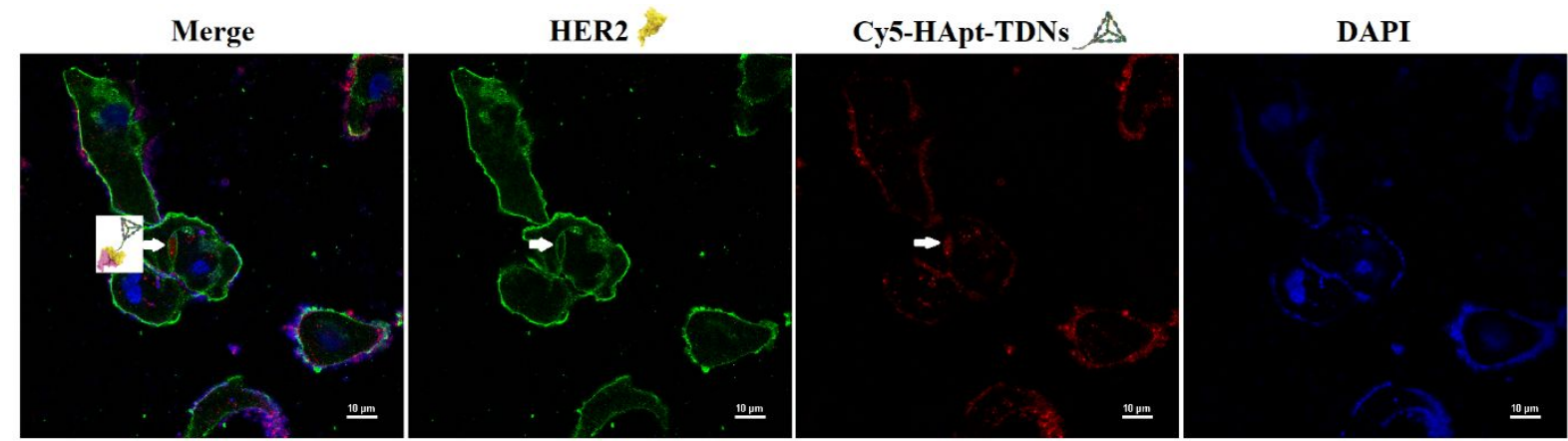

Figure S16. The Observation HER2-Cy5-HApt-tFNA Complex (white arrow: HER2-Cy5HApt-tFNA complex, HER2: green, Cy5-HApt-tFNA: red, nucleus: blue). Scale bars are 10 $\mu \mathrm{m}$.

\section{Supporting information for videos.}

Video S1. SK-BR-3 cells were treated with 250 nM Cy5-HApt-tFNA for 12 h. (HER2: green, Cy5-HApt-tFNA: red, nucleus: blue).

Video S2. SK-BR-3 cells were treated with 250 nM Cy5-HApt-tFNA for 12 h. (HER2: green, Cy5-HApt-tFNA: red, nucleus: blue).

Video S3. SK-BR-3 cells were treated with 250 nM Cy5-HApt-tFNA for 24 h. (HER2: green, Cy5-HApt-tFNA: red, nucleus: blue).

Video S4. SK-BR-3 cells were treated with 250 nM Cy5-HApt-tFNA for 24 h. (HER2: green, Cy5-HApt-tFNA: red, nucleus: blue). 


\section{References}

2 (1) Han, Y. P.; Li, X. M.; Chen, H. B.; Hu, X. J.; Luo, Y.; Wang, T.; Wang, Z. J.; Li, Q.; Fan, C. H.; Shi,

3 J. Y.; Wang, L. H.; Zhao, Y.; Wu, C. F.; Chen, N. Real-Time Imaging of Endocytosis and Intracellular

4 Trafficking of Semiconducting Polymer Dots. ACS App/ Mater Interfaces. 2017, 9, 21200-21208.

5 (2) Ma, W.; Shao, X.; Zhao, D.; Li, Q.; Liu, M.; Zhou, T.; Xie, X.; Mao, C.; Zhang, Y.; Lin, Y. Self-

6 Assembled Tetrahedral DNA Nanostructures Promote Neural Stem Cell Proliferation and Neuronal

7 Differentiation. ACS App/ Mater Interfaces. 2018, 10, 7892-7900.

8 (3) Ma, W. J.; Xie, X. P.; Shao, X. R.; Zhang, Y. X.; Mao, C. C.; Zhan, Y. X.; Zhao, D.; Liu, M. T.; Li,

9 Q. S.; Lin, Y. F. Tetrahedral DNA nanostructures facilitate neural stem cell migration via activating 10 RHOA/ROCK2 signalling pathway. Cell Prolif. 2018, 51.

11 (4) Shao, X. R.; Ma, W. J.; Xie, X. P.; Li, Q. S.; Lin, S. Y.; Zhang, T.; Lin, Y. F. Neuroprotective Effect 12 of Tetrahedral DNA Nanostructures in a Cell Model of Alzheimer's Disease. ACS App/Mater Interfaces. $2018,10,23682-23692$.

14 (5) Shi, S.; Peng, Q.; Shao, X.; Xie, J.; Lin, S.; Zhang, T.; Li, Q.; Li, X.; Lin, Y. Self-Assembled 15 Tetrahedral DNA Nanostructures Promote Adipose-Derived Stem Cell Migration via IncRNA XLOC 16010623 and RHOA/ROCK2 Signal Pathway. ACS Appl Mater Interfaces. 2016, 8, 19353-63.

17 (6) Shi, S. R.; Lin, S. Y.; Li, Y.; Zhang, T.; Shao, X. R.; Tian, T. R.; Zhou, T. F.; Li, Q. S.; Lin, Y. F. 18 Effects of tetrahedral DNA nanostructures on autophagy in chondrocytes. Chem Commun. 2018, 54, $19 \quad 1327-1330$.

20 (7) Tian, T. R.; Zhang, T.; Zhou, T. F.; Lin, S. Y.; Shi, S. R.; Lin, Y. F. Synthesis of an ethyleneimine/tetrahedral DNA nanostructure complex and its potential application as a multi-functional delivery vehicle. Nanoscale. 2017, 9, 18402-18412. 
1 (8) Zhang, Y. X.; Ma, W. J.; Zhu, Y.; Shi, S. R.; Li, Q. S.; Mao, C. C.; Zhao, D.; Zhan, Y. X.; Shi, J. Y.;

2 Li, W.; Wang, L. H.; Fan, C. H.; Lin, Y. F. Inhibiting Methicillin-Resistant Staphylococcus aureus by 3 Tetrahedral DNA Nanostructure-Enabled Antisense Peptide Nucleic Acid Delivery. Nano letters. 2018, $4 \quad 18,5652-5659$. 